\title{
Clinical and biomarker changes in sporadic Alzheimer's disease
}

Junjie Zhuo Ph.D. ${ }^{1,2}$; Yuanchao Zhang Ph.D. ${ }^{5}$; Bing Liu Ph.D. ${ }^{1,3,4}$; Yong Liu Ph.D. ${ }^{1,34}$; Xiaoqing Zhou Ph.D. ${ }^{2}$; Perry

F. Bartlett Ph.D. ${ }^{2 *} ;$ Tianzi Jiang Ph.D. ${ }^{1-5 *}$; and the Alzheimer's Disease Neuroimaging Initiative

${ }^{1}$ Brainnetome Center, Institute of Automation, Chinese Academy of Sciences, Beijing 100190, China

${ }^{2}$ The Queensland Brain Institute, University of Queensland, Brisbane, QLD 4072, Australia

${ }^{3}$ University of Chinese Academy of Sciences, Beijing 100049, China

${ }^{4}$ CAS Center for Excellence in Brain Science, Institute of Automation, Chinese Academy of Sciences, Beijing 100190, China

${ }^{5}$ The Clinical Hospital of Chengdu Brain Science Institute, MOE Key Lab for Neuroinformation, University of Electronic Science and Technology of China, Chengdu 625014, China

*Tianzi Jiang and Perry F. Bartlett are the corresponding authors.

\section{Group Information:}

*Data used in preparation of this article were obtained from the Alzheimer's Disease Neuroimaging Initiative (ADNI) database (adni.loni.usc.edu). As such, the investigators within the ADNI contributed to the design and implementation of ADNI and/or provided data but did not participate in analysis or writing of this report. A complete listing of ADNI investigators can be found at: http://adni.loni.usc.edu/wp

content/uploads/how_to_apply/ADNI_Acknowledgement_List.pdf.

\section{Corresponding author:}

Tianzi Jiang, PhD, Brainnetome Center, Institute of Automation, Chinese Academy of Sciences, Beijing 100190, China (Phone: +86-1-82544778, Fax: +86-1 82544777, Email: jiangtz@nlpr.ia.ac.cn) Perry F. Bartlett, PhD, Queensland Brain Institute, The University of Queensland, Qld, 4072, Australia (Phone: +617 3346-6311, Email: p.bartlett@uq.edu.au) 


\section{Key Points}

Question: What is the role of amyloid- $\beta$ in dynamic changes in biomarkers and clinical profiles in the progression of sporadic Alzheimer's disease?

Findings: The changes of the hippocampal volume and FDG that were consistent with the changes of the clinical profiles showed a non-linear change in the initial stage and an accelerated non-linear change during MCI2AD, changes in amyloid biomarkers were inconsistent with the clinical profile. Cognitively normal people with elevated or normal amyloid showed no significant differences in clinical measures, hippocampal volume, or FDG.

Meaning: Amyloid- $\beta$ alone may not be used as the central index for defining the preclinical stage of SAD. 


\section{Abstract}

IMPORTANCE: The dynamic changes of biomarkers and clinical profiles in sporadic Alzheimer's disease (SAD) are poorly understood.

OBJECTIVE: To evaluate the impact of amyloid- $\beta(A \beta)$ biomarkers on SAD by measuring the dynamic changes in biomarkers and clinical profiles in the progression of SAD.

DESIGN AND SETTING: This retrospective and longitudinal study analyzed clinical and biomarker data from 665 participants (mean follow-up $4.90 \pm 2.83$ years) from a subset of the AD Neuroimaging Initiative (ADNI) participants collected from August 2005 to December 2018. By aligning the timing of the changes in the various biomarkers with the stable normal cognition $(\mathrm{CN})$ baseline and mild cognitive impairment $(\mathrm{MCI})$ or $\mathrm{AD}$ onset timepoints, we combined data from the stable $\mathrm{CN}, \mathrm{CN}$ conversion to $\mathrm{MCI}(\mathrm{CN} 2 \mathrm{MCI})$, and $\mathrm{MCI}$ conversion to $\mathrm{AD}(\mathrm{MCI} 2 \mathrm{AD})$ groups to identify the trajectories associated with the progression of $\mathrm{AD}$.

PARTICIPANTS: The participants were 294 CN, 69 CN2MCI, 300 MCI2AD, and 24 who converted from CN to $\mathrm{MCI}$ to $\mathrm{AD}(\mathrm{CN} 2 \mathrm{MCI} 2 \mathrm{AD})$ (of whom 22 were also included in the $\mathrm{CN} 2 \mathrm{MCI}$ ).

EXPOSURES: Amyloid- $\beta$ measured by florbetapir positron emission tomography (PET) or cerebrospinal fluid assay of amyloid- $\beta\left(\operatorname{CSF} A \beta_{42}\right)$.

MAIN OUTCOMES AND MEASURES: The measures included the 13-item cognitive subscale of the AD Assessment Scale (ADAS13, as a clinical measure), hippocampal volume, and the fluorodeoxyglucose (FDG) PET standardized uptake value ratio (SUVR).

RESULTS: The CN, CN2MCI, and MCI2AD subgroups' median (interquartile range [IQR]) annual changes in ADAS13 were (0.388 [-0.278, 0.818], 1.000 [0.239, 2.330], and 3.388 [1.750, 6.169]). The annual changes 
in hippocampal volume for each group were $(-0.005 \% \mathrm{ICV}[-0.011,-0.001],-0.006 \% \mathrm{ICV}[-0.012,-0.002]$, and $-0.014 \%$ ICV $[-0.021,-0.009])$. The annual changes in FDG PET SUVR for each group were $(-0.011[-0.030$, $0.010],-0.027[-0.056,-0.012]$, and $-0.039[-0.063,0.014])$. Changes in the amyloid biomarkers were inconsistent with clinical profile changes. The annual changes in CSF A $\beta_{42}$ for each group were $(-1.500 \mathrm{pg} / \mathrm{ml}$ [-6.000, 4.000], -2.200 [-5.667, 4.000], and -2.000 [-7.000, 2.650]) and in A $\beta$ PET SUVR for each group were (0.004 [-0.002, 0.012], $0.004[-0.001,0.011]$, and $0.005[-0.006,0.014])$. During the stable CN and CN2MCI stages, subjects with elevated and those with normal amyloid showed no significant differences (likelihood ratio test, $p<.01)$ in clinical measures, hippocampal volume, or FDG.

CONCLUSIONS AND RELEVANCE: Hippocampal volume and FDG associated with clinical profiles impairment in the SAD progression. $\mathrm{A} \beta$ alone is not associated with clinical profiles, hippocampal volume, and FDG impairment in the preclinical stage of SAD. 


\section{Introduction}

2 The diagnostic guidelines for Alzheimer's disease (AD) ${ }^{1,2}$ provide a clinical-pathological framework. The

3 National Institute on Aging-Alzheimer's Association (NIA-AA), in line with the amyloid hypothesis ${ }^{3,4}$, defines

$4 \mathrm{AD}$ on the basis of biomarkers, rather than by clinical symptoms 5 . However, two observations, the failure of all

5 anti-amyloid- $\beta(\mathrm{A} \beta)$ drugs $^{6-9}$ to show clinical efficacy and the discovery that amyloid plaques are not unique to

$6 \mathrm{AD}^{5,10}$, have led to a debate about the central role of amyloid in the etiology of the disease and its usefulness as

7 a diagnostic marker of AD.

8 To address this debate, identifying which dynamic changes in biomarkers and clinical profiles correlate directly

with the progression of $\mathrm{AD}$ is essential. The relevant studies have primarily relied on patients with autosomal

dominant $\mathrm{AD}$ (ADAD), who often have a predictable age at onset ${ }^{11-13}$. In contrast, the precise timing of the

disease for patients with sporadic $\mathrm{AD}(\mathrm{SAD})$ is difficult to predict ${ }^{14}$. Because the ADAD genetic mutations (APP,

PSEN1, and PSEN2) cause alterations in $\mathrm{A} \beta$ processing, $\mathrm{ADAD}$ studies have consistently found that $\mathrm{A} \beta$ is the

first and key biomarker, followed by changes in other biomarkers and clinical profiles ${ }^{11-13}$. However, increasing

evidence has shown that patients with SAD are associated with multiple gene factors, which affect more than

$\mathrm{A} \beta$ processing ${ }^{10,15-17}$. Since $\mathrm{ADAD}$ only accounts for a very small proportion (approximately $1 \%$ ) of $\mathrm{AD}^{11}$, how

widely applicable the findings obtained from $\mathrm{ADAD}$ to SAD remains a question ${ }^{10}$.

A previous prospective $\mathrm{SAD}$ study based the stage of $\mathrm{AD}$ on the level of accumulation of amyloid and found, consistent with $\mathrm{ADAD}$ studies, that the $\mathrm{A} \beta$ abnormality appeared first, followed by other changes ${ }^{18}$. However, due to its assumption that $A \beta$ is the etiological agent, that study does not consider the possible dynamic biomarker and clinical changes which occur in relation to symptom onset as in the previous ADAD studies ${ }^{11-13}$.

21 Even in subjects who have over 15 years of longitudinal data, the baseline has not been aligned with the onset 
of clinical symptom to investigate longitudinal changes in biomarkers and clinical profiles ${ }^{19}$. However, as the progression of $\mathrm{AD}$ has been hypothesized to be non-linear ${ }^{20,21}$, simply aligning the baseline with $\mathrm{A} \beta$ levels or studying the longitude data is not sufficient to chart the progression of SAD. Thus, to reduce this limitation, we aligned the timepoints of the clinical diagnosis of mild cognitive impairment (MCI) or AD onset to investigate

\section{Methods}

\section{Study design}

The data were obtained from the ADNI dataset (http://adni.loni.usc.edu/) and downloaded in December 2018.

The ADNI was launched in 2003 as a public-private partnership, led by Principal Investigator Michael W.

Weiner, MD. The primary goal of ADNI has been to test whether serial magnetic resonance imaging (MRI), positron emission tomography (PET), other

biological markers, and clinical and neuropsychological assessment can be combined to measure the progression of MCI and early AD.

To estimate the timing, order, and trajectory of clinical and biomarker changes from normal aging to $\mathrm{AD}$, we labeled the data of the three subgroups as $\mathrm{CN}$, subjects with normal cognition who were confirmed to convert to $\mathrm{MCI}(\mathrm{CN} 2 \mathrm{MCI})$, and subjects with $\mathrm{MCI}$ who were confirmed to convert to $\mathrm{AD}(\mathrm{MCI} 2 \mathrm{AD})$. The $\mathrm{CN}$ subgroup was defined as either subjects who had a cognitively normal baseline, showed no significant memory concern (SMC), and had at least two years' follow-up without conversion to MCI or AD or as subjects with a baseline of MCI who reversed to $\mathrm{CN}$ within one year and remained stable $\mathrm{CN}$ for at least 2 years to the end of follow- 
subsequent diagnosis of having converted to MCI in the follow-up or as subjects with a SMC confirmed as having converted to MCI. To increase the sample size and statistical power, the CN2MCI timepoint of subjects who converted to $\mathrm{MCI}$ and finally to $\mathrm{AD}$ were also included in the CN2MCI group. The MCI2AD subgroup was defined as subjects with a baseline diagnosis of MCI who converted to stable AD in the follow-up.

To precisely reflect the stage of disease, we selected those subjects within the CN2MCI and MCI2AD subgroups who had one year or less between the initial one-time assessment before the disease onset and the disease onset of MCI or AD.

\section{Assessments}

The clinical profiles and biomarkers used in the present study included the 13-item cognitive subscale of the

Alzheimer's Disease Assessment Scale (ADAS13), Mini-Mental State Examination (MMSE), Clinical

Dementia Rating Scale-Sum of Boxes (CDRSB), hippocampal volumes, fluorodeoxyglucose (FDG) positron emission tomography (PET), florbetapir PET, and CSF biomarkers (including tau, phosphor-tau (Ptau), and $A \beta_{42}$ ). FDG and florbetapir PET, were measured by the standardized uptake value ratio (SUVR). Please see the Supplementary materials for all the metadata downloaded from the ADNI dataset and the detailed assessment of each clinical profile and biomarker.

Participants were categorized into elevated amyloid or normal amyloid subsets depending on their florbetapir SUVR or CSF $A \beta_{42}$ status. Elevated amyloid was defined as a florbetapir SUVR greater than $0.79^{22}$ or a CSF $\mathrm{A} \beta_{42}$ value less than $192 \mathrm{pg} / \mathrm{mL}^{23}$. Participants were classified as having elevated amyloid if they met the cutoff threshold at any timepoint. Otherwise, they were classified as having normal amyloid. If there was no amyloid

61 information for a participant, their data were classified as missing. 
As the ADAS has usually been used to monitor the progression of $\mathrm{AD}^{8,24}$, we calculated the correlations between the ADAS13 and each marker in the CN2MCI and MCI2AD stages separately to evaluate whether the markers could predict $\mathrm{AD}$ progression.

To compare the progression curve for all the markers and verify the model of the fitted results, the scaled value for each marker was defined by (raw data - mean CN baseline value) / the standard deviation (SD) of the whole dataset. To further verify the abnormal pattern of the markers in the progression of $\mathrm{AD}$, we also analyzed the within-individual trajectories for all 24 subjects who were initially diagnosed as $\mathrm{CN}$, subsequently converted to MCI, and then to AD (CN2MCI2AD). Each marker in these individuals was also scaled by the mean of the baseline data for the $\mathrm{CN}$ subgroup and for the SD of the entire dataset.

\section{Statistical analysis}

72 For the longitudinal trajectory analyses of the CN2MCI and MCI2AD subgroups, the follow-up years were categorized into pre-symptom onset ( $<0$ onset years) and post-symptom onset ( $>0$ onset years). To increase model convergence, we excluded the data of timepoints for which the sample size was less than 3 for each clinical profile or biomarker, (See Fig. S1 for the detailed sample size for the various timepoints for each clinical profile or biomarker) Statistical analyses and plotting were performed using R (version 3.5.3, https://www.rproject.org/)

Longitudinal trajectory models were constructed for the various biomarkers using linear mixed effects models ${ }^{25}$. For each marker, we started by fitting an appropriate function to the time (baseline or onset time) e.g. time + time $^{\wedge 2}+$ time $^{\wedge 3}$. Disease progression $(\mathrm{CN}, \mathrm{CN} 2 \mathrm{MCI}$, and MCI2AD) was included in the models to extract

81 disease-specific biomarker trajectories. Covariates such as age at baseline or onset year, sex, APOE\&4, and education were included as confounds, and a backward elimination method was used for model selection. We 
then selected a structure for the random effects and covariance structure for the residuals in the model. All the model selections were based on the Akaike Information Criterion ${ }^{14,26}$, an objective model selection tool.

Maximum likelihood was used to fit the mixed-effect models as it is robust to the absence of random data ${ }^{27}$.

We further compared the trajectories for each marker in the progression of $A D$ to uncover differences between the elevated amyloid and normal amyloid groups. The overall amyloid effect was tested using likelihood ratio tests that compared the full model to a reduced model with no amyloid factor in each subgroup for each marker.

For any subgroup that showed a significant amyloid effect as the disease progressed, a supplementary post hoc analysis was performed between the elevated amyloid and normal amyloid groups at each timepoint based on the estimated marginal means derived from the model.

To determine the timing of the dysfunctions, we fitted a linear mixed effects model to the CN2MCI subgroup with time as a categorical variable for each biomarker. The post hoc analysis was conducted between each timepoint based on estimated marginal means derived from the model.

\section{Results}

Of the downloaded 665 subjects from the ADNI dataset, we utilized the data from 663 participants in the group analysis (CN: 294, CN2MCI: 69, and MCI2AD: 300, for more details of the participants' characteristics see

Table 1) and $24 \mathrm{CN} 2 \mathrm{MCI} 2 \mathrm{AD}$ participants in the individual analysis (the data from the group of 22 participants in the $\mathrm{CN} 2 \mathrm{MCI}$ stage was combined into the above CN2MCI group analysis). Some participants were followed for up to 13 years with a mean follow-up period of $4.90 \pm 2.83$ years.

Figure 1 shows the trajectories of the biomarkers estimated by the linear mixed effects models across groups 
the hippocampal volume and FDG levels remained stable throughout the CN stage followed by slow, non-linear

changes in the CN2MCI stage and rapid non-linear changes in the MCI2AD. Even the values of the florbetapir

PET and the CSF biomarkers were normal in $\mathrm{CN}$, intermediate in CN2MCI and abnormal in MCI2AD

subgroups, the shape of the trajectories for florbetapir PET and the CSF biomarkers did not consistent with the

were $(0.388[-0.278,0.818], 1.000[0.239,2.330]$, and $3.388[1.750,6.169], p<.001$, respectively). The annual

$p<.001$, respectively). The annual changes in MMSE for each group were $(0.000[-0.250,0.161],-0.286[-$

$0.571,0.000]$, and $-1.500[-2.775,-0.800], p<.001$, respectively). The annual changes in hippocampal volume

for each group were $(-0.005 \% \mathrm{ICV}[-0.011,-0.001],-0.006 \% \mathrm{ICV}[-0.012,-0.002]$, and $-0.014 \% \mathrm{ICV}[-0.021$,

Florbetapir PET SUVR for each group were $(0.004$ [-0.002, 0.012], 0.004 [-0.001,0.011], and 0.005 [-0.006,

0.014], $p=.840$, respectively). The annual changes in CSF A $\beta_{42}$ for each group were $(-1.500 \mathrm{pg} / \mathrm{ml}[-6.000$, tau for each group were $(0.775 \mathrm{pg} / \mathrm{ml}[-1.887,4.500], 2.150[-0.500,7.900]$, and $3.000[-3.900,14.175], p=.121$, respectively). The annual changes in CSF ptau for each group were $(1.050 \mathrm{pg} / \mathrm{ml}[-1.450,4.500], 1.980[-0.200$, 5.050], and $1.408[-1.321,8.325], p=.628$, respectively).

Figure 2 shows the trajectories of the biomarker changes with either the normal or elevated amyloid groups. 
ended with a phase in which rapid non-linear changes appeared in the MCI2AD. We found no significant differences in the clinical profiles, hippocampal volume, or FDG changes between the elevated and normal amyloid subjects at the $p<.05$ level. The statistical results showed no difference for CDRSB and FDG in any of the three (CN, CN2MCI, and MCI2AD) subgroups at $p<.05$. The ADAS13 analysis showed significant group differences for the 6-9-year time period in the $\mathrm{CN}$ subgroup, for the $<-4.5$ and $>4$ years to onset time in the CN2MCI subgroup, and for the $>-0.5$ years to onset time in the MCI2AD subgroup at $p<.05$. The MMSE analysis showed a significant group difference for the time period $>-1$ year in the CN2MCI subgroup at $p<.05$. Although the likelihood ratio test showed a significant difference in hippocampal volume between the elevated and normal amyloid subjects ( $p=.047)$, the post-hoc results showed no significance at $p<.05$ in the CN2MCI and only showed a significant group difference for the time period $<-1$ years in the MCI2AD at $p<.05$. All subgroups showed obvious significant differences with respect to florbetapir PET and CSF A $\beta_{42}$ between the elevated and normal amyloid subjects at $p<.001$. For CSF Tau and CSF Ptau, only the CN2MCI subgroup showed no amyloid effect at $p<.05$; the other two subgroups showed significant differences at $p<.05$ (Figure 2; for the post hoc analysis results, see eTables 10-15).

We found that the changes in the CDRSB (Figure 3.1A and 2A), MMSE (Figure 3.1B and 3.2B), hippocampal volume (Figure 3.1C and 3.2C), and FDG PET in the post-cingulate cortex (Figure 3.1D and 3.2D) were associated with the change in the ADAS13 in both the CN2MCI and MCI2AD subgroups. However, the changes in the amyloid related biomarkers florbetapir PET and CSF A $\beta_{42}$ were not significantly associated with the change in the ADAS13 in either group (Figure 3.1 E, 3.1F, 3.2 E, and 3.2 F).

Combining the biomarker findings, we assessed the trajectories and order of pathophysiological changes for the clinical, imaging, and biochemical measures (Figure 4.A and 4.B). As can be seen in Figures 1 and 2, the clinical 
profiles, hippocampal volume, and FDG changed slowly in the initial stage of CN2MCI and accelerated in the late MCI2AD stage. The order in which these measures changed in the CN2MCI subgroup was that the hippocampus and FDG PET changed earlier than ADAS13 and that CDRSB and MMSE were the last measures to change. Further, a post hoc analysis showed that the change in hippocampal volume preceded the symptom onset of MCI by 2.5 years and ADAS13 preceded the symptom onset of MCI by 1 year. Significant changes in

MMSE and CDRSB were concurrent with MCI onset (Supplement Figure 3). Even in patients with elevated amyloid, the trajectory of the amyloid-related biomarker was not consistent with the clinical profiles, hippocampal volume, or FDG (Figure 4.B). More importantly, florbetapir PET was stable during the CN2MCI stage. Although CSF A $\beta_{42}$ showed some nonlinear changes before MCI onset, the change was smaller than those of the other biomarkers. Thus, these results do not support previous reports ${ }^{11,12}$, suggesting that amyloid-related biomarker changes largely lead other biomarker changes at the onset of the disease.

We further assessed each biomarker for the individuals who progressed from $\mathrm{CN}$ to $\mathrm{MCI}$ and to $\mathrm{AD}$ for each 1-7) years in these 24 subjects. The individual results were consistent with the previous group results: The trajectories of their clinical profiles changed slowly in the initial period in the CN2MCI stage and accelerated in the MCI2AD stage, the dynamic changes of hippocampal volume paralleled the disease status changes, and there were no significant changes in amyloid-related biomarkers in the $\mathrm{CN}$ to $\mathrm{MCI}$ to $\mathrm{AD}$ progression.

\section{Discussion}

163 Identifying the dynamic changes in clinical assessments and biomarkers during a patient's progression to AD is critical for defining the stage of the disease and its etiology and for monitoring the efficacy of potential therapies. 
timepoints of the different stages from $\mathrm{CN}$, through $\mathrm{MCI}$, to $\mathrm{AD}$ using various clinical assessments and

biomarkers to obtain a panorama of disease progression. The end stage of $\mathrm{CN}$ remained stable during the follow-

up. Furthermore, the onset timepoints of clinical diagnosis were rigidly aligned for CN2MCI and MCI2AD with

error less than 1 year. In addition, we also replicated the group results using the CN2MCI2AD subjects. Thus,

the study expands the current literature about the SAD clinical and biomarker dynamic changes.

171 We found that the accumulation of amyloid in the $\mathrm{CN}$ did not predict future cognitive impairment in either 172 people who maintained a stable $\mathrm{CN}$ or those in the pre-MCI onset stage (Figure 2.A-C). This result is consistent 173 with recent reports that indicated that brain $A \beta$ is not clinically relevant ${ }^{28,29}$. Other studies, however, reported 174 that elevated amyloid in $\mathrm{CN}$ individuals was associated with a higher likelihood of cognitive decline compared 175 with normal amyloid $\mathrm{CN}$ subjects ${ }^{27,30}$. Although these findings are insightful, using the same ADNI dataset, we 176 found that cognitive decline did not depend on the accumulation of amyloid but on the clinical stage of the 177 disease. Moreover, our results were partially consistent with previous findings, showing that amyloid 178 accumulation alone cannot predict the cognitive decline or disease progression, whereas the amyloid 179 accumulation combined with tau or atrophy of some groups can predict the further cognitive decline ${ }^{31-33}$ or 180 disease progression in the normal adults ${ }^{34-36}$. These results support the recent idea about the combination 181 therapies for future $\mathrm{AD}$ treatment strategies ${ }^{37}$ and the widespread concern about overdiagnosis in the preclinical $182 \mathrm{AD}^{38}$

183 By aligning the disease onset timepoint, we found that dynamic changes in amyloid-related biomarkers were not associated with a change in disease status even in elevated amyloid subjects (Figures 2.F, 2.G, 4.B, 4.C). A previous prospective study, based on the amyloid hypothesis, reported that brain $\mathrm{A} \beta$ deposition continuously changed with SAD progression ${ }^{18}$. However, they found that the raw data of $A \beta$ deposition was stable and 

to $\mathrm{MCI}$ to $\mathrm{AD}$, we found that the trajectory of hippocampal volume and FDG were consistent with the clinical profiles in that they did not follow a sigmoid curve ${ }^{20,21}$ but rather showed a slow change in the initial stage and accelerated changes in the later stage from MCI to AD (Figure 4). Although previous studies based on the ADNI dataset reported that the changes in these biomarkers followed a sigmoid curve $\mathrm{e}^{39-41}$, these studies did not align their findings with the stage of disease, so they could not be considered to accurately reflect the trajectory of biomarker changes that occur in the progression of $\mathrm{AD}$. Thus, these results indicated that assessment of the clinical or biomarker dynamic changes by aligning the clinical onset timepoint maybe better reflect and characterize the progress of the disease.

Our finding that cognitive decline and $A \beta$ deposition did not occur in parallel (Figures 3 and 4 ) is consistent with previous studies that reported that $\mathrm{A} \beta$ dysregulation poorly correlates with $\mathrm{AD}$ severity ${ }^{42}$, progressive neurodegeneration ${ }^{43}$, cognitive dysfunction ${ }^{44}$, or brain atrophy ${ }^{45}$. During the rapid cognitive decline from MCI to $\mathrm{AD}, \mathrm{A} \beta$ deposition only mildly increased. This may partially explain why anti-A $\beta$ drugs have failed in clinical trials. Medications, such as solanezumab, a medication designed to clear soluble $A \beta$ from the brain, are used in the mild AD stage ${ }^{8}$, which is too late to prevent rapid cognitive decline. In addition, ADAS13 showed dramatic changes about 1 year before the clinical MCI onset, a finding which was not consistent with the general concept that clinical profiles change only after the onset of $\mathrm{MCI}^{20,21}$. Thus, the slow stage from pre-MCI to pre-AD may be a better time window for future clinical trial design.

Our results suggest that applying ADAD results directly to SAD research may not be appropriate ${ }^{10}$. We found that the rate of $A \beta$ biomarker changes during $\mathrm{CN}$ conversion to MCI stage did not reflect those of other 
208 ADAD studies that found that amyloid biomarkers undergo greater changes and lead to other biomarker changes 209 in the initial stage of symptom onset ${ }^{11-13}$. The most likely explanation for this difference is that the ADAD and 210 SAD have different etiologies ${ }^{10}$. In addition, we found that dramatic hippocampal atrophy starts 2.5 years prior 211 to MCI onset, which is later than recent ADAD brain atrophy findings ${ }^{13,14}$. The concept that SAD involves a 212 long pre-symptomatic period and is derived from ADAD studies ${ }^{15}$ may need to be reconsidered.

\section{Limitations}

214 One of the limitations of the current study is that the CN2MCI subgroup was older than the MCI2AD subgroup, 215 which may have influenced the pattern of biomarker changes. The ongoing ADNI dataset maybe resolve this 216 limitation in future studies. Another limitation is the small sample size of the tau and A $\beta$ biomarkers in the pre217 MCI stage, which meant that we could not fully reveal the dynamic changes in these biomarkers in the 218 preclinical stage. The ongoing collection of plasma biomarkers ${ }^{46-50}$ and ADNI3 tau-related PET data ${ }^{51}$ will 219 improve the likelihood of fully understanding the preclinical stage of SAD in the future.

\section{Conclusions}

221 Hippocampal volume and FDG associated with clinical profiles impairment in the SAD progression. A $\beta$ alone 222 is not associated with clinical profiles, hippocampal volume, and FDG impairment in the preclinical stage of 223 SAD.

Author Contributions: T.J. and P.F.B. supervised the study. J.Z., P.F.B., and T.J. were responsible for the design of the concept and the study. J.Z. contribution to the data analysis and statistical analysis; Y.Z., B.L., Y.L., and X.Z. made substantial contributions to the discussion on the results and the manuscript; J.Z., P.F.B., and T.J. wrote the manuscript. 
Acknowledgments: This work was partially supported by the Natural Science Foundation of China (Grant Nos.

31620103905 and 81701781), the Science Frontier Program of the Chinese Academy of Sciences (Grant No.

QYZDJ-SSW-SMC019), the Guangdong Pearl River Talents Plan (2016ZT06S220), and the International

Postdoctoral Exchange Fellowship Program 2017 by the Office of China Postdoctoral Council.

Data collection and sharing for this project was funded by the Alzheimer's Disease Neuroimaging Initiative

(ADNI) (National Institutes of Health Grant U01 AG024904) and DOD ADNI (Department of Defense award

number W81XWH-12-2-0012). ADNI is funded by the National Institute on Aging, the National Institute of

Biomedical Imaging and Bioengineering, and through generous contributions from the following: AbbVie,

Alzheimer's Association; Alzheimer's Drug Discovery Foundation; Araclon Biotech; BioClinica, Inc.; Biogen;

Bristol-Myers Squibb Company; CereSpir, Inc.; Cogstate; Eisai Inc.; Elan Pharmaceuticals, Inc.; Eli Lilly and

Company; EuroImmun; F. Hoffmann-La Roche Ltd and its affiliated company Genentech, Inc.; Fujirebio; GE

Healthcare; IXICO Ltd.; Janssen Alzheimer Immunotherapy Research \& Development, LLC.; Johnson \&

Johnson Pharmaceutical Research \& Development LLC.; Lumosity; Lundbeck; Merck \& Co., Inc.; Meso Scale

Diagnostics, LLC.; NeuroRx Research; Neurotrack Technologies; Novartis Pharmaceuticals Corporation; Pfizer

Inc.; Piramal Imaging; Servier; Takeda Pharmaceutical Company; and Transition Therapeutics. The Canadian

Institutes of Health Research is providing funds to support ADNI clinical sites in Canada. Private sector contributions are facilitated by the Foundation for the National Institutes of Health (www.fnih.org). The grantee organization is the Northern California Institute for Research and Education, and the study is coordinated by the Alzheimer's Therapeutic Research Institute at the University of Southern California. ADNI data are disseminated by the Laboratory for Neuro Imaging at the University of Southern California. 
249 Fan who offered constructive comments, and Dr. Rhoda E., Edmund F. Perozzi, and Rowan Tweedale for their

editing assistance and discussions.

Conflict of interest: The authors declare that they have no competing financial interests.

\section{References}

1. McKhann GM, Knopman DS, Chertkow H, et al. The diagnosis of dementia due to Alzheimer's disease: recommendations from the National Institute on Aging-Alzheimer's Association workgroups on diagnostic guidelines for Alzheimer's disease. Alzheimers Dement. 2011;7(3):263-269.

2. Dubois B, Feldman HH, Jacova C, et al. Advancing research diagnostic criteria for Alzheimer's disease: the IWG-2 criteria. Lancet Neurol. 2014;13(6):614-629.

3. Hardy JA, Higgins GA. Alzheimer's disease: the amyloid cascade hypothesis. Science. 1992;256(5054):184185.

4. Selkoe DJ, Hardy J. The amyloid hypothesis of Alzheimer's disease at 25 years. EMBO Mol Med. 2016;8(6):595-608.

5. Jack CR, Jr., Bennett DA, Blennow K, et al. NIA-AA Research Framework: Toward a biological definition of Alzheimer's disease. Alzheimers Dement. 2018;14(4):535-562.

6. Panza F, Lozupone M, Seripa D, Imbimbo BP. Amyloid-beta immunotherapy for alzheimer disease: Is it now a long shot? Ann Neurol. 2019;85(3):303-315.

7. Panza F, Lozupone M, Logroscino G, Imbimbo BP. A critical appraisal of amyloid-beta-targeting therapies for Alzheimer disease. Nat Rev Neurol. 2019;15(2):73-88.

8. Honig LS, Vellas B, Woodward M, et al. Trial of Solanezumab for Mild Dementia Due to Alzheimer's Disease. N Eng/ J Med. 2018;378(4):321-330.

9. Wessels AM, Tariot PN, Zimmer JA, et al. Efficacy and Safety of Lanabecestat for Treatment of Early and Mild Alzheimer Disease: The AMARANTH and DAYBREAK-ALZ Randomized Clinical Trials. JAMA Neurol. 2019.

10. Morris GP, Clark IA, Vissel B. Questions concerning the role of amyloid-beta in the definition, aetiology and diagnosis of Alzheimer's disease. Acta Neuropathol. 2018;136(5):663-689.

11. Bateman RJ, Xiong C, Benzinger TL, et al. Clinical and biomarker changes in dominantly inherited Alzheimer's disease. N Eng/ J Med. 2012;367(9):795-804.

12. Yau WW, Tudorascu DL, McDade EM, et al. Longitudinal assessment of neuroimaging and clinical markers in autosomal dominant Alzheimer's disease: a prospective cohort study. Lancet Neurol. 2015;14(8):804813.

13. McDade E, Wang G, Gordon BA, et al. Longitudinal cognitive and biomarker changes in dominantly inherited Alzheimer disease. Neurology. 2018;91(14):e1295-e1306.

14. Gordon BA, Blazey TM, Su Y, et al. Spatial patterns of neuroimaging biomarker change in individuals from families with autosomal dominant Alzheimer's disease: a longitudinal study. Lancet Neurol. 2018;17(3):241250.

15. Ryan NS, Rossor MN, Fox NC. Alzheimer's disease in the 100 years since Alzheimer's death. Brain. 2015;138(Pt 12):3816-3821. 
16. Jansen IE, Savage JE, Watanabe K, et al. Genome-wide meta-analysis identifies new loci and functional pathways influencing Alzheimer's disease risk. Nature Genetics. 2019;51(3):404-413.

17. Heart CF, Genetic, Ad ERI, Genetic PD, Perades. Genetic meta-analysis of diagnosed Alzheimer's disease identifies new risk loci and implicates $A \beta$, tau, immunity and lipid processing. Nature Genetics. 2019;51(3):414-430.

18. Villemagne VL, Burnham S, Bourgeat $\mathrm{P}$, et al. Amyloid beta deposition, neurodegeneration, and cognitive decline in sporadic Alzheimer's disease: a prospective cohort study. Lancet Neurol. 2013;12(4):357-367.

19. Veitch DP, Weiner MW, Aisen PS, et al. Understanding disease progression and improving Alzheimer's disease clinical trials: Recent highlights from the Alzheimer's Disease Neuroimaging Initiative. Alzheimers Dement. 2019;15(1):106-152.

20. Jack CR, Jr., Knopman DS, Jagust WJ, et al. Tracking pathophysiological processes in Alzheimer's disease: an updated hypothetical model of dynamic biomarkers. Lancet Neurol. 2013;12(2):207-216.

21. Jack CR, Jr., Knopman DS, Jagust WJ, et al. Hypothetical model of dynamic biomarkers of the Alzheimer's pathological cascade. Lancet Neurol. 2010;9(1):119-128.

22. Landau SM, Horng A, Jagust WJ, Alzheimer's Disease Neuroimaging I. Memory decline accompanies subthreshold amyloid accumulation. Neurology. 2018;90(17):e1452-e1460.

23. Shaw LM, Vanderstichele H, Knapik-Czajka M, et al. Cerebrospinal fluid biomarker signature in Alzheimer's disease neuroimaging initiative subjects. Ann Neurol. 2009;65(4):403-413.

24. Egan MF, Kost J, Tariot PN, et al. Randomized Trial of Verubecestat for Mild-to-Moderate Alzheimer's Disease. N Eng/ J Med. 2018;378(18):1691-1703.

25. West BT, Welch KB, Galecki AT. Linear mixed models: a practical guide using statistical software. Chapman and Hall/CRC; 2014.

26. Akaike H. Information theory and an extension of the maximum likelihood principle. In: Selected papers of hirotugu akaike. Springer; 1998:199-213.

27. Donohue MC, Sperling RA, Petersen R, et al. Association Between Elevated Brain Amyloid and Subsequent Cognitive Decline Among Cognitively Normal Persons. JAMA. 2017;317(22):2305-2316.

28. Jansen WJ, Ossenkoppele R, Tijms BM, et al. Association of Cerebral Amyloid-beta Aggregation With Cognitive Functioning in Persons Without Dementia. JAMA PSychiatry. 2018;75(1):84-95.

29. Dubois B, Epelbaum $S$, Nyasse $F$, et al. Cognitive and neuroimaging features and brain $\beta$-amyloidosis in individuals at risk of Alzheimer's disease (INSIGHT-preAD): a longitudinal observational study. Lancet Neurol. 2018;17(4):335-346.

30. Insel PS, Hansson O, Mackin RS, Weiner M, Mattsson N, Alzheimer's Disease Neuroimaging I. Amyloid pathology in the progression to mild cognitive impairment. Neurobiol Aging. 2018;64:76-84.

31. Jack CR, Jr., Wiste HJ, Therneau TM, et al. Associations of Amyloid, Tau, and Neurodegeneration Biomarker Profiles With Rates of Memory Decline Among Individuals Without Dementia. JAMA. 2019;321(23):23162325.

32. Zhao Y, Tudorascu DL, Lopez OL, et al. Amyloid beta Deposition and Suspected Non-Alzheimer Pathophysiology and Cognitive Decline Patterns for 12 Years in Oldest Old Participants Without Dementia. JAMA Neurol. 2018;75(1):88-96.

33. Bilgel M, An Y, Helphrey J, et al. Effects of amyloid pathology and neurodegeneration on cognitive change in cognitively normal adults. Brain. 2018;141(8):2475-2485.

34. Vos SJ, Verhey F, Frolich L, et al. Prevalence and prognosis of Alzheimer's disease at the mild cognitive impairment stage. Brain. 2015;138(Pt 5):1327-1338.

35. Vos SJ, Xiong C, Visser PJ, et al. Preclinical Alzheimer's disease and its outcome: a longitudinal cohort study. 
Lancet Neurol. 2013;12(10):957-965.

36. Burnham SC, Bourgeat $\mathrm{P}$, Doré $\mathrm{V}$, et al. Clinical and cognitive trajectories in cognitively healthy elderly individuals with suspected non-Alzheimer's disease pathophysiology (SNAP) or Alzheimer's disease pathology: a longitudinal study. Lancet Neurol. 2016;15(10):1044-1053.

37. Long JM, Holtzman DM. Alzheimer Disease: An Update on Pathobiology and Treatment Strategies. Cell. 2019;179(2):312-339.

38. Langa KM, Burke JF. Preclinical Alzheimer Disease-Early Diagnosis or Overdiagnosis? JAMA Intern Med. 2019.

39. Caroli A, Frisoni GB, Alzheimer's Disease Neuroimaging I. The dynamics of Alzheimer's disease biomarkers in the Alzheimer's Disease Neuroimaging Initiative cohort. Neurobiol Aging. 2010;31(8):1263-1274.

40. Schuff N, Tosun D, Insel PS, et al. Nonlinear time course of brain volume loss in cognitively normal and impaired elders. Neurobiol Aging. 2012;33(5):845-855.

41. Jack CR, Jr., Vemuri P, Wiste HJ, et al. Shapes of the trajectories of 5 major biomarkers of Alzheimer disease. Arch Neurol. 2012;69(7):856-867.

42. Arriagada PV, Growdon JH, Hedley-Whyte ET, Hyman BT. Neurofibrillary tangles but not senile plaques parallel duration and severity of Alzheimer's disease. Neurology. 1992;42(3):631-631.

43. Holmes $C$, Boche $D$, Wilkinson $D$, et al. Long-term effects of $A \beta 42$ immunisation in Alzheimer's disease: follow-up of a randomised, placebo-controlled phase I trial. The Lancet. 2008;372(9634):216-223.

44. Giannakopoulos P, Herrmann F, Bussière T, et al. Tangle and neuron numbers, but not amyloid load, predict cognitive status in Alzheimer's disease. Neurology. 2003;60(9):1495-1500.

45. Jack CR, Jr., Lowe VJ, Weigand SD, et al. Serial PIB and MRI in normal, mild cognitive impairment and Alzheimer's disease: implications for sequence of pathological events in Alzheimer's disease. Brain 2009;132(Pt 5):1355-1365.

46. Nakamura A, Kaneko N, Villemagne VL, et al. High performance plasma amyloid- $\beta$ biomarkers for Alzheimer's disease. Nature. 2018;554(7691):249-254.

47. Janelidze S, Mattsson N, Palmqvist S, et al. Plasma P-tau181 in Alzheimer's disease: relationship to other biomarkers, differential diagnosis, neuropathology and longitudinal progression to Alzheimer's dementia. Nat Med. 2020;26(3):379-386.

48. Thijssen EH, La Joie R, Wolf A, et al. Diagnostic value of plasma phosphorylated tau181 in Alzheimer's disease and frontotemporal lobar degeneration. Nat Med. 2020;26(3):387-397.

49. Ashton NJ, Hye A, Rajkumar AP, et al. An update on blood-based biomarkers for non-Alzheimer neurodegenerative disorders. Nat Rev Neurol. 2020;16(5):265-284.

50. Mattsson N, Cullen NC, Andreasson U, Zetterberg H, Blennow K. Association Between Longitudinal Plasma Neurofilament Light and Neurodegeneration in Patients With Alzheimer Disease. JAMA Neurol. 2019;76(7):791-799.

51. Scholl M, Lockhart SN, Schonhaut DR, et al. PET Imaging of Tau Deposition in the Aging Human Brain. Neuron. 2016;89(5):971-982. 


\section{Figure legends}

\section{Figure 1. Estimated group trajectories of clinical profiles and biomarkers}

(A) ADAS13; range from 0 [best] to 85 [worst], (B) CDRSB; range from 0 [best] to 18 [worst], (C) MMSE; range from 0 [worst] to 30 [best], (D) the MRI measures of hippocampal volumes adjusted by percent of the total intracranial volume (ICV), (E) the post-cingulate cortex glucose metabolism measured by fluorodeoxyglucose (FDG) positron emission tomography (PET) consistently showed stable changes in the stable cognitive normal $(\mathrm{CN})$ subgroup, slow non-linear changes in the confirmed $\mathrm{CN}$ conversion to $\mathrm{MCI}$ (CN2MCI) subgroup, and acceleration non-linear changes in the confirmed MCI conversion to AD (MCI2AD) subgroup. In contrast, (F) Florbetapir PET, (G) CSF A $\beta_{42}$, (H) CSF tau, and (I) CSF phosphor-tau (Ptau) did not show changes consistent with the clinical profiles.

The estimated trajectory and 95\% confidence interval from the linear mixed models (yellow line and yellow shaded area, respectively) are plotted against years from baseline or symptom (MCI or AD) onset for each marker. The black dashed line represents the MCI onset timepoint. The red dashed line represents the AD onset timepoint.

Figure 2. Estimated elevated and normal amyloid group trajectories of clinical profiles and biomarkers See Figure 1 for explanation of each panel sub-title.

The estimated trajectory and 95\% confidence interval from the linear mixed models are plotted against years from baseline or symptom (MCI or $\mathrm{AD}$ ) onset for each marker. Red line and pink shaded area represent the elevated amyloid subjects. Blue line and blue shaded area represent the normal amyloid subjects.

L.R.= likelihood ratio

Figure 3. Relationship between the change in each biomarker and the change in ADAS13 in the CN conversion to MCI and the MCI conversion to AD subgroups

The top panels show that the changes in the (1.A) CDRSB score, (1.B) MMSE score, (1.C) hippocampal volume percent of ICV, and (1.D) post-cingulate FDG SUVR value significantly correlated with the change in the ADAS13 scores in the $\mathrm{CN}$ conversion to MCI subgroup. However, the changes in the amyloid-related biomarkers, (1.E) Florbetapir PET SUVR and (1.F) CSF A $\beta_{42}$, were not significantly correlated with the change in ADAS13 scores.

The bottom panels show that the change in the (2.A) CDRSB score, (2.B) MMSE score, (2.C) hippocampal volume percent of ICV, and (2.D) post-cingulate FDG SUVR value significantly correlated with the change in ADAS13 scores in the MCI conversion to AD subgroup. However, the changes in amyloid related biomarkers, (2.E) Florbetapir PET SUVR and (2.F) CSF A $\beta_{42}$, were not significantly correlated with the change in ADAS13 scores.

$\mathrm{df}=$ degree of freedom

\section{Figure 4. Temporal evolution of marker changes and within-individual trajectories of marker changes} Raw data for each biomarker and clinical profile converted to scaled values. The scaled value for each marker was defined by: (raw data - mean $\mathrm{CN}$ baseline value) / the standard deviation of the whole dataset.

(A) Clinical profiles, hippocampal volume, and FDG scaled changes in all subjects;

(B) Clinical profiles and biomarkers scaled changes in the elevated amyloid subjects;

Clinical profiles and biomarkers scaled changes in 4 subjects who included the entire disease process from $\mathrm{CN}$ conversion to MCI followed by conversion to $\mathrm{AD}$ (CN2MCI2AD), within-individual changes. 


\section{Table}

\section{Table 1. Characteristics of Study Participants}

414 Abbreviations: ADASA13, the 13-item cognitive subscale of the Alzheimer's Disease Assessment Scale; 415 CDRSB, the Clinical Dementia Rating Scale-Sum of Boxes; MMSE, the Mini-Mental State Examination; ICV, 416 intracranial volume; FDG, fluorodeoxyglucose; CSF, cerebrospinal fluid; SUVR, standardized uptake value 417 ratio; SD, standard deviation

\begin{tabular}{|c|c|c|c|c|}
\hline & $\mathrm{CN}$ & $\mathrm{CN} 2 \mathrm{MCl}$ & MCI2AD & $p$ \\
\hline & $N=294$ & $\mathrm{~N}=69$ (22 finally to $A D$ ) & $N=300$ & \\
\hline Sex: & & & & .058 \\
\hline Female & $146(49.7 \%)$ & $28(40.6 \%)$ & $121(40.3 \%)$ & \\
\hline Male & $148(50.3 \%)$ & $41(59.4 \%)$ & $179(59.7 \%)$ & \\
\hline Education, mean (SD), y & $16.5(2.68)$ & $16.1(2.67)$ & $15.9(2.80)$ & .032 \\
\hline APOE allele: & & & & $<.001$ \\
\hline APOE\&4 noncarriers & $221(75.2 \%)$ & $42(60.9 \%)$ & $98(32.7 \%)$ & \\
\hline APOE\&4 carriers & $73(24.8 \%)$ & 27 (39.1\%) & $202(67.3 \%)$ & \\
\hline Follow-up, mean (SD), y & $5.33(2.83)$ & $5.70(3.43)$ & $4.07(2.29)$ & $<.001$ \\
\hline Amyloid characteristics: & & & & $<.001$ \\
\hline Missing amyloid information & $52(17.7 \%)$ & $8(11.6 \%)$ & $75(25.0 \%)$ & \\
\hline Elevated amyloid & 115 (39.1\%) & $41(59.4 \%)$ & $202(67.3 \%)$ & \\
\hline Normal Amyloid & $127(43.2 \%)$ & $20(29.0 \%)$ & $23(7.7 \%)$ & \\
\hline & $\begin{array}{c}\text { Baseline } \\
\text { characteristics }\end{array}$ & $\begin{array}{c}\mathrm{MCl} \text { onset } \\
\text { characteristics }\end{array}$ & $\begin{array}{c}\text { AD onset } \\
\text { characteristics }\end{array}$ & \\
\hline Age, mean (SD), y & $74.0(6.18)$ & $79.7(5.70)$ & 76.5 (7.39) & $<.001$ \\
\hline ADAS13, mean (SD) & $8.60(4.04)$ & $15.0(6.32)$ & $27.1(7.36)$ & $<.001$ \\
\hline CDRSB, mean (SD) & $0.06(0.26)$ & $1.01(0.76)$ & $4.30(1.59)$ & $<.001$ \\
\hline MMSE, mean (SD) & $29.1(1.17)$ & $27.6(1.83)$ & $23.8(2.91)$ & $<.001$ \\
\hline Hippocampal volume, mean (SD), \%ICV & $0.50(0.06)$ & $0.44(0.05)$ & $0.37(0.06)$ & $<.001$ \\
\hline FDG PET SUVR, mean (SD) & $1.41(0.14)$ & $1.20(0.15)$ & $1.16(0.14)$ & $<.001$ \\
\hline Amyloid PET SUVR, mean (SD) & $0.78(0.09)$ & $0.93(0.14)$ & $1.01(0.12)$ & $<.001$ \\
\hline CSF A $\beta 42$, mean (SD), pg/ml & $207(49.9)$ & $194(79.0)$ & $137(35.6)$ & $<.001$ \\
\hline CSF tau, mean (SD), pg/ml & $63.8(29.2)$ & $90.4(28.8)$ & $130(75.9)$ & $<.001$ \\
\hline CSF ptau, mean (SD), pg/ml & $29.1(14.7)$ & $39.2(23.7)$ & $55.1(30.7)$ & $<.001$ \\
\hline
\end{tabular}




\section{Hippocampal volume}

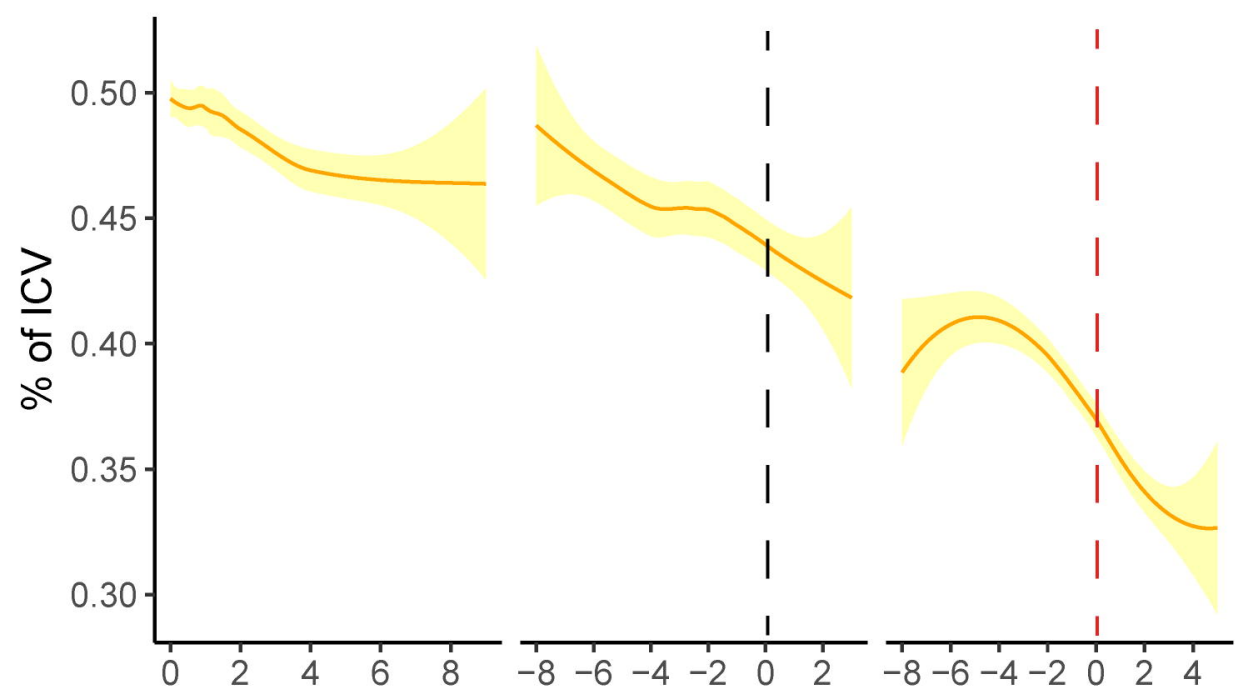

\section{G CSF AB42}

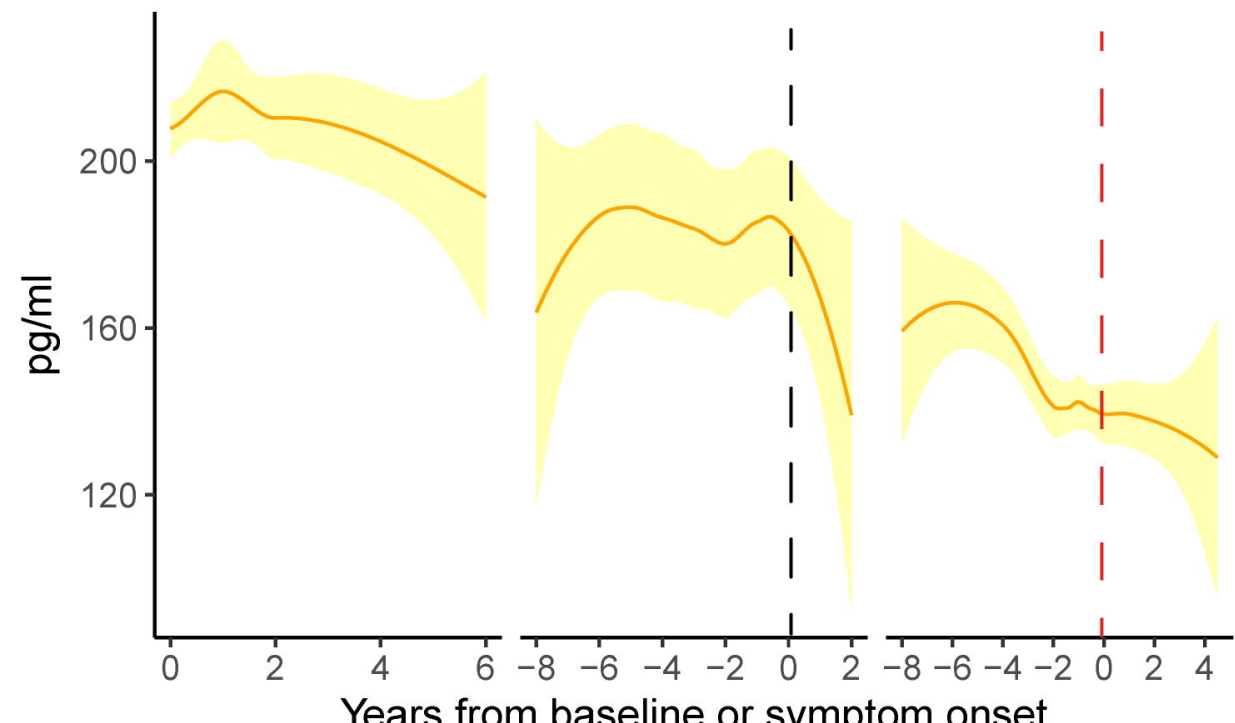

\section{E FDG-Postcingulate}

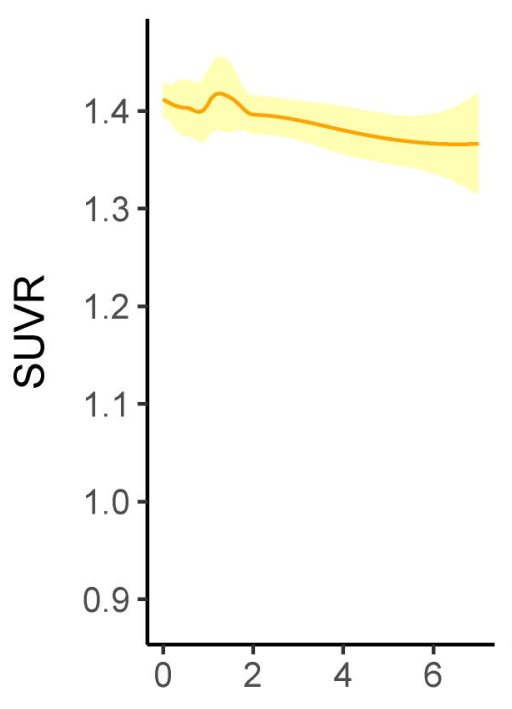

\section{H CSF Tau}

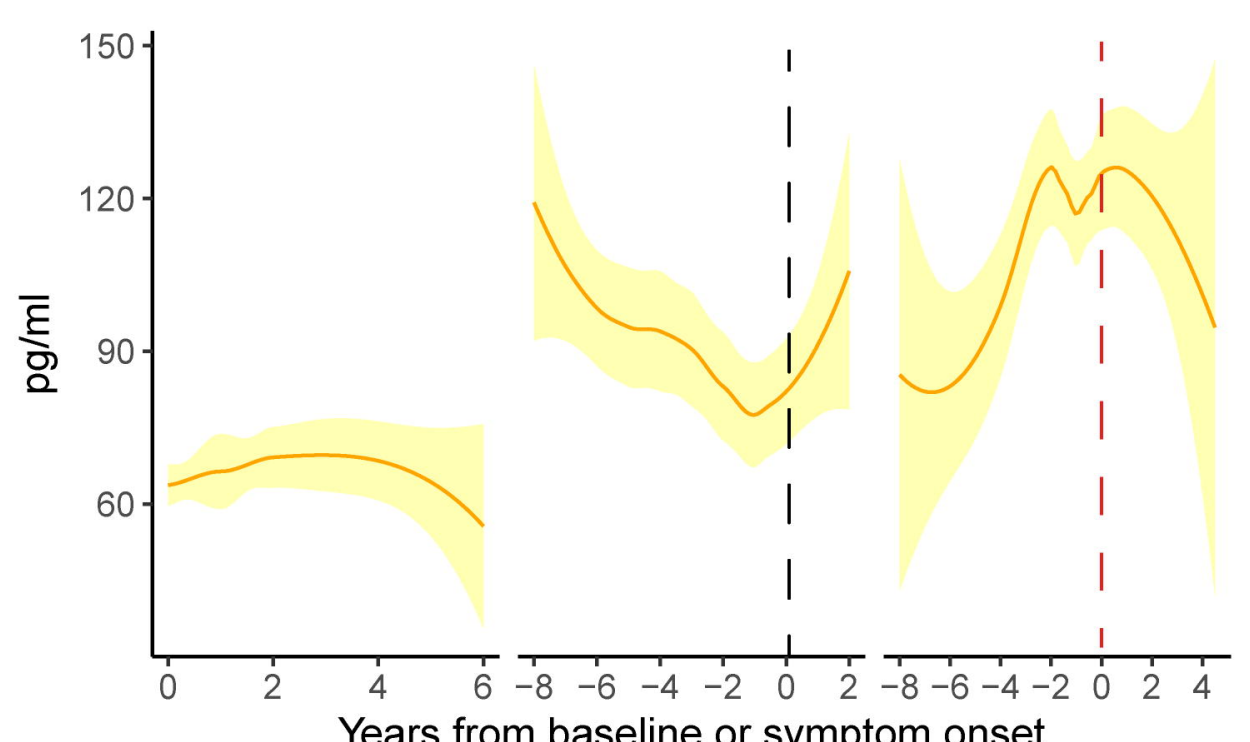

\section{F Florbetapir PET}
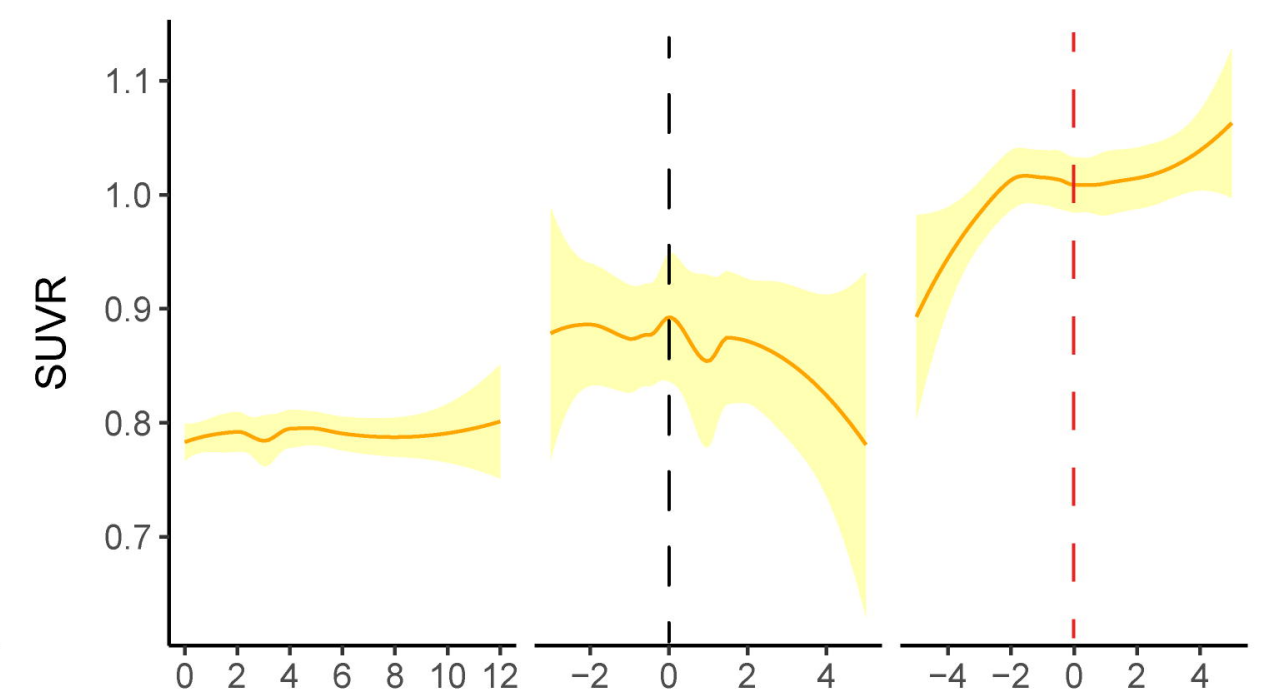

I CSF Ptau

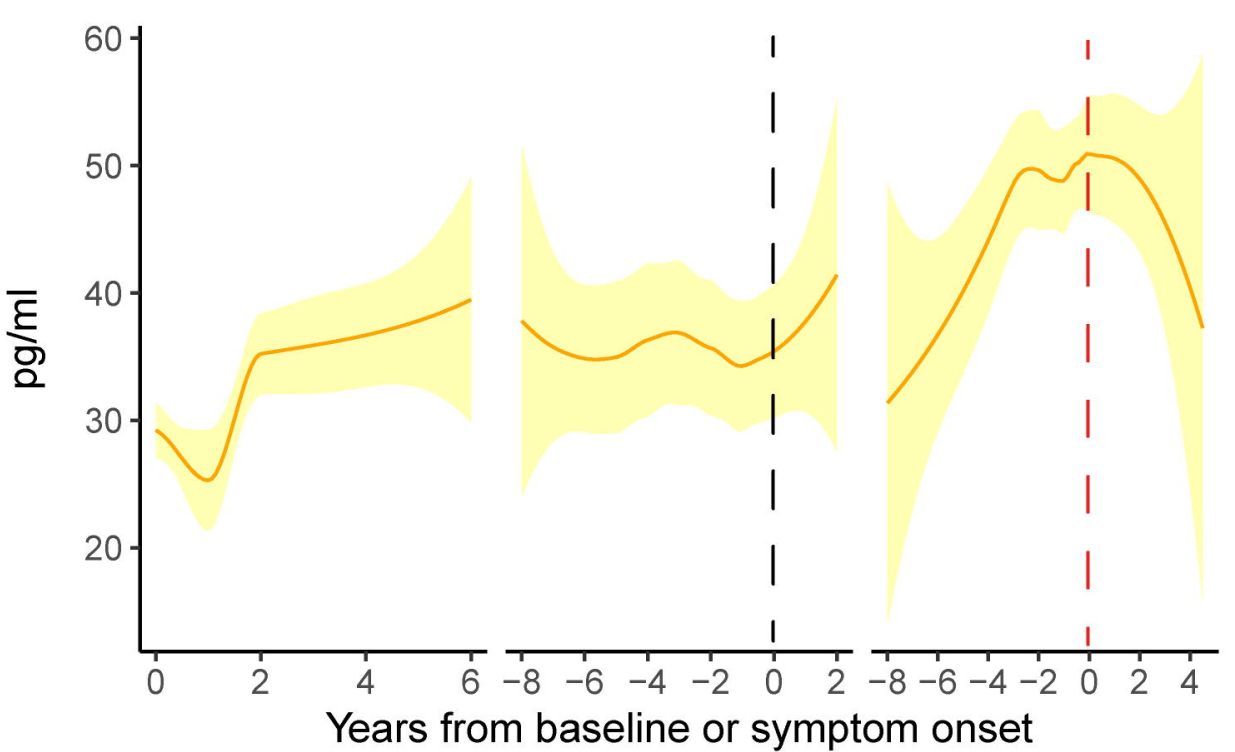



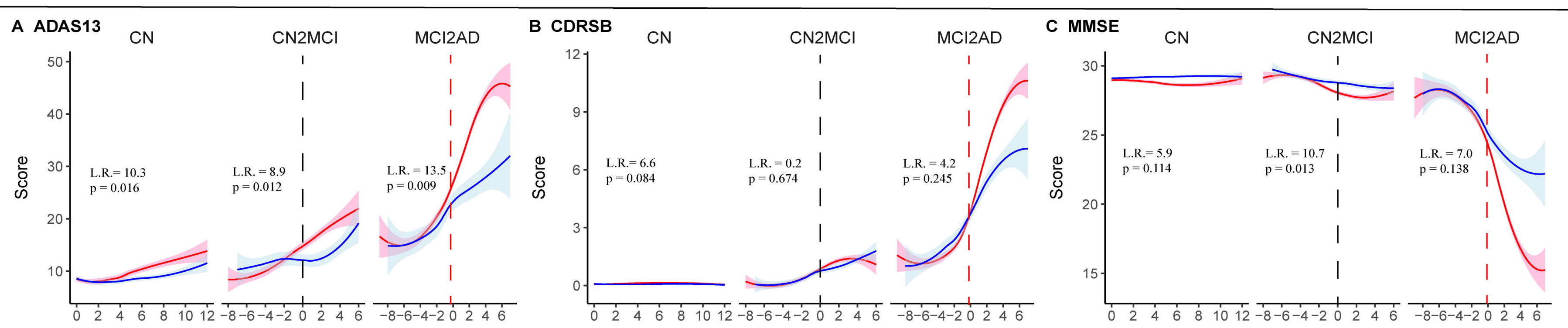

\section{Hippocampal volume}
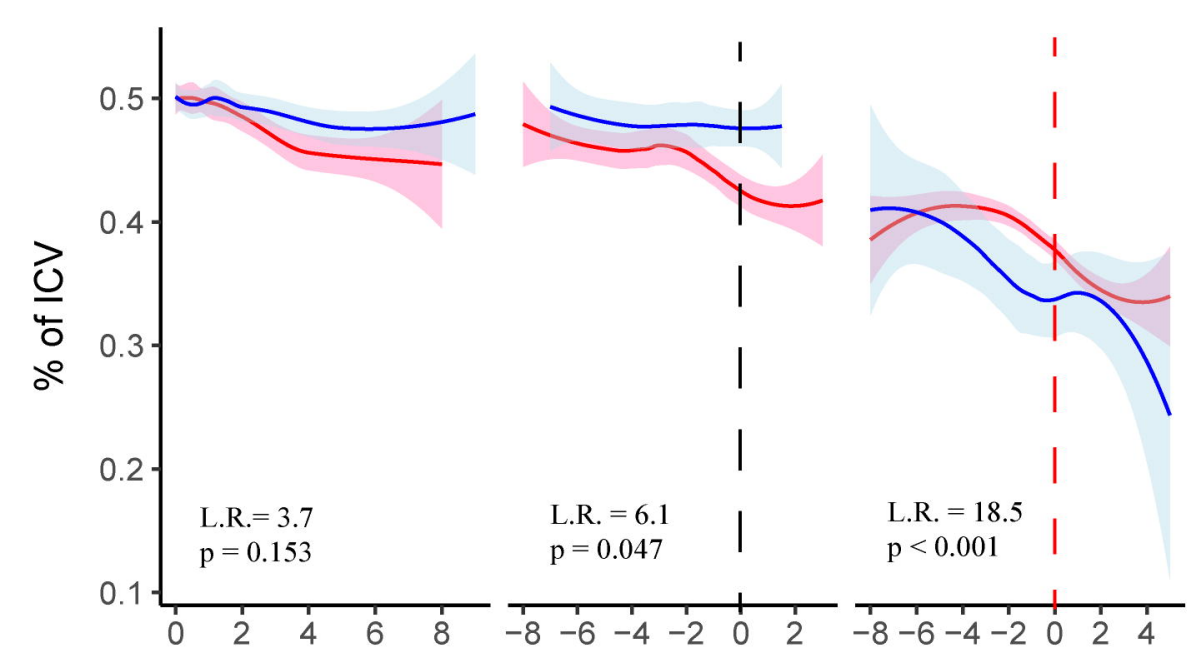

G CSF Aß42

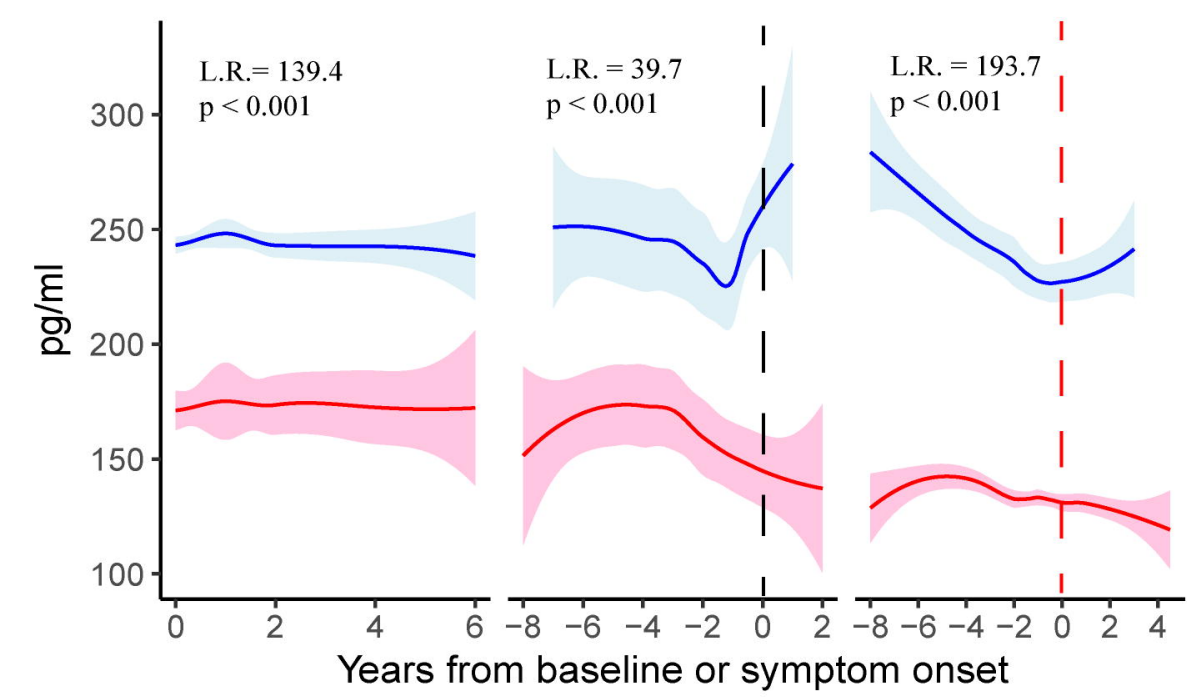

\section{E FDG-Postcingulate}

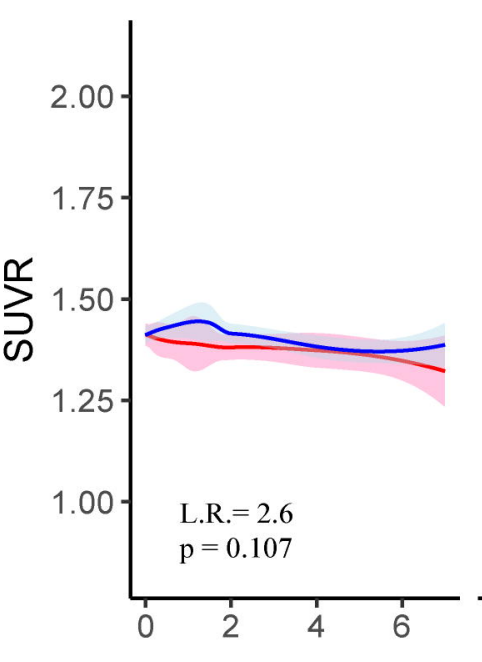

\section{H CSF Tau}

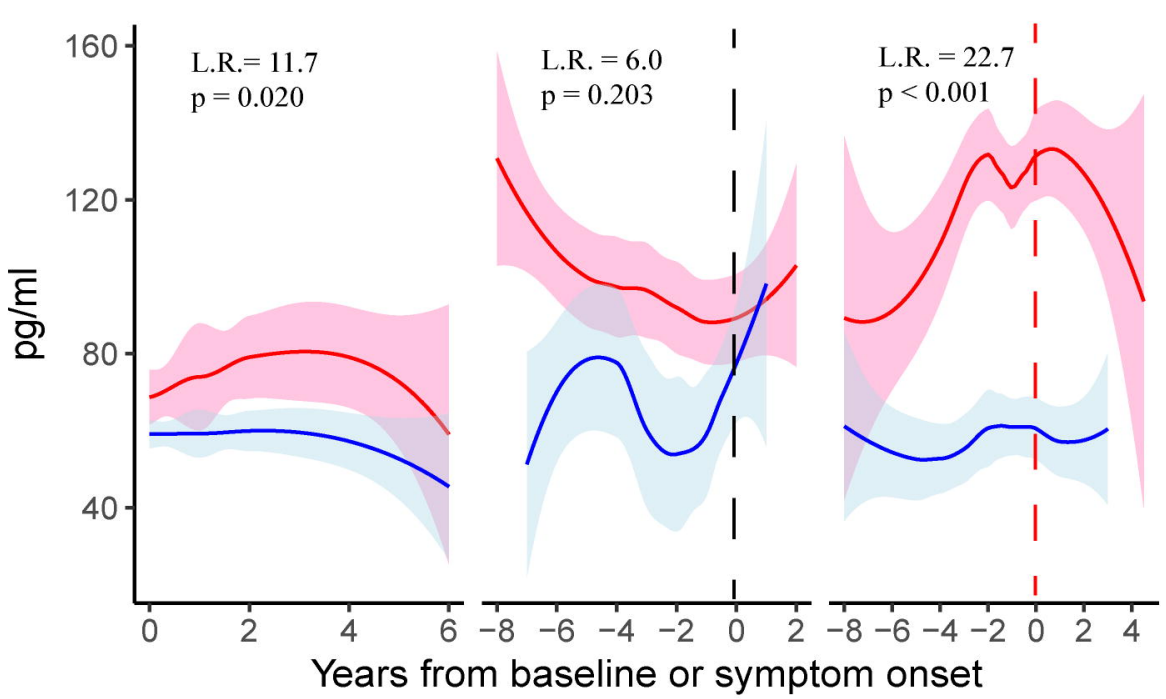

\section{F Florbetapir PET}

$$
\sum_{\infty}^{\infty}
$$
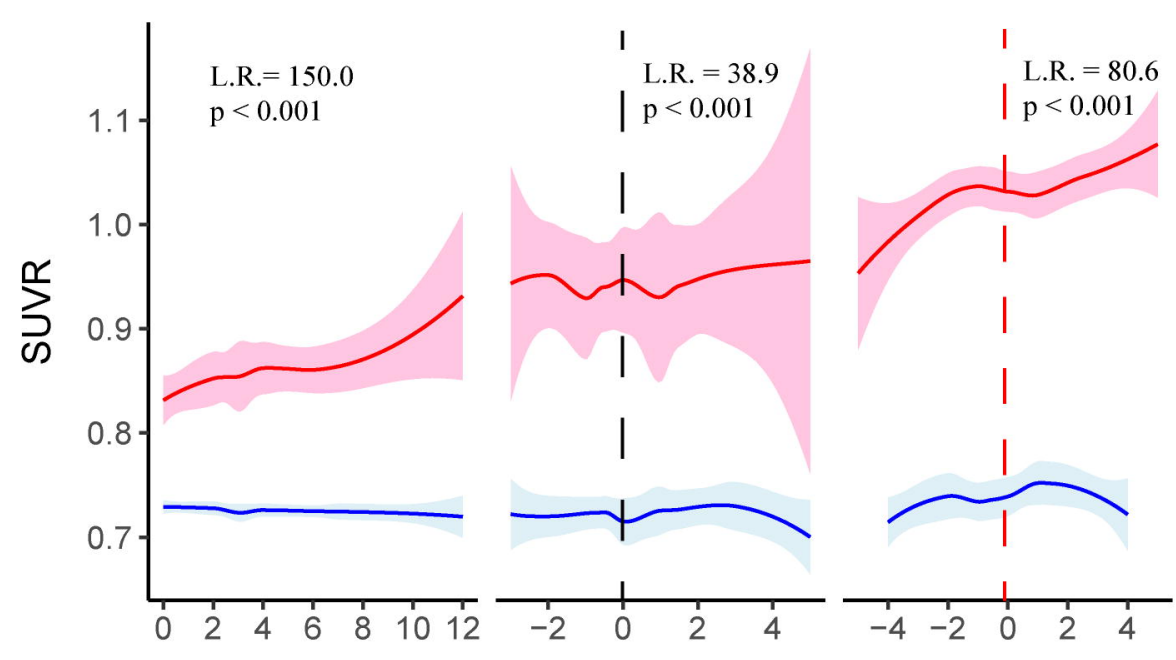

\section{CSF Ptau}

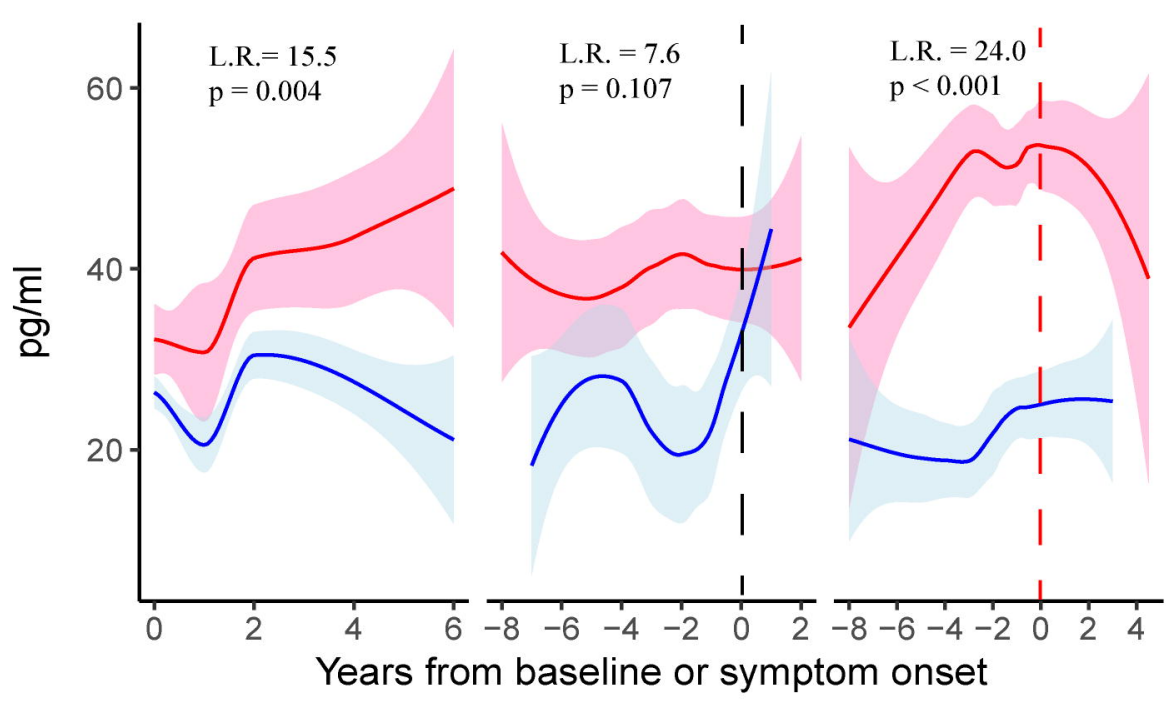



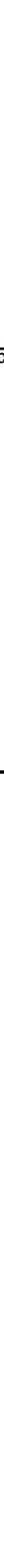

$\mathrm{MCl}$ Convert to $\mathrm{AD}$

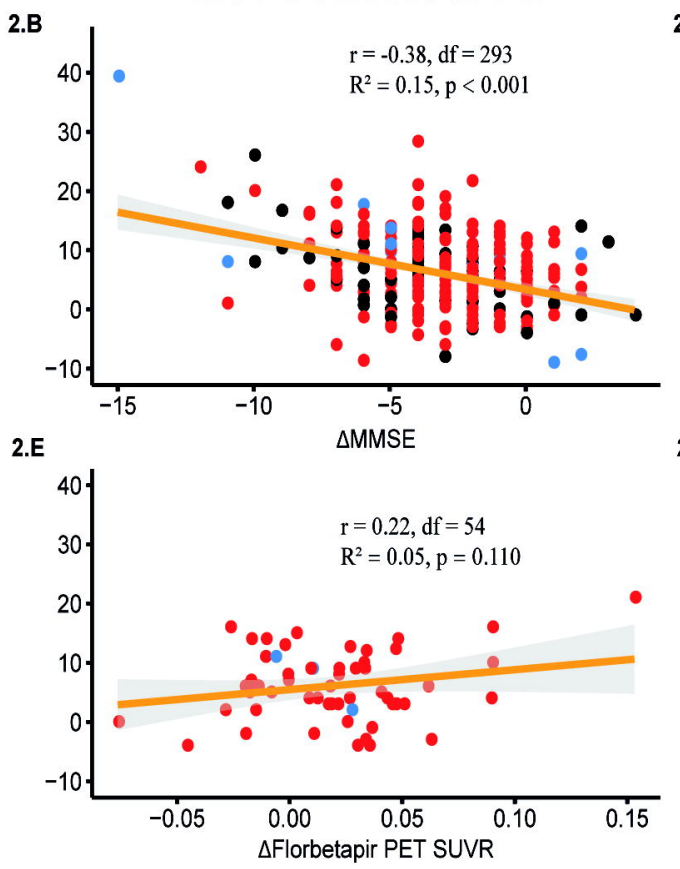

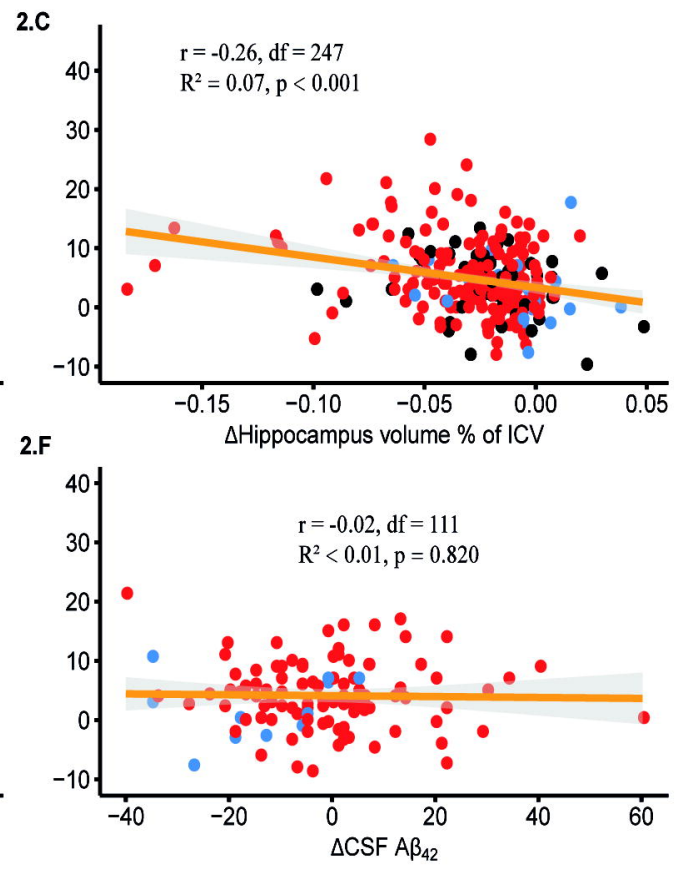




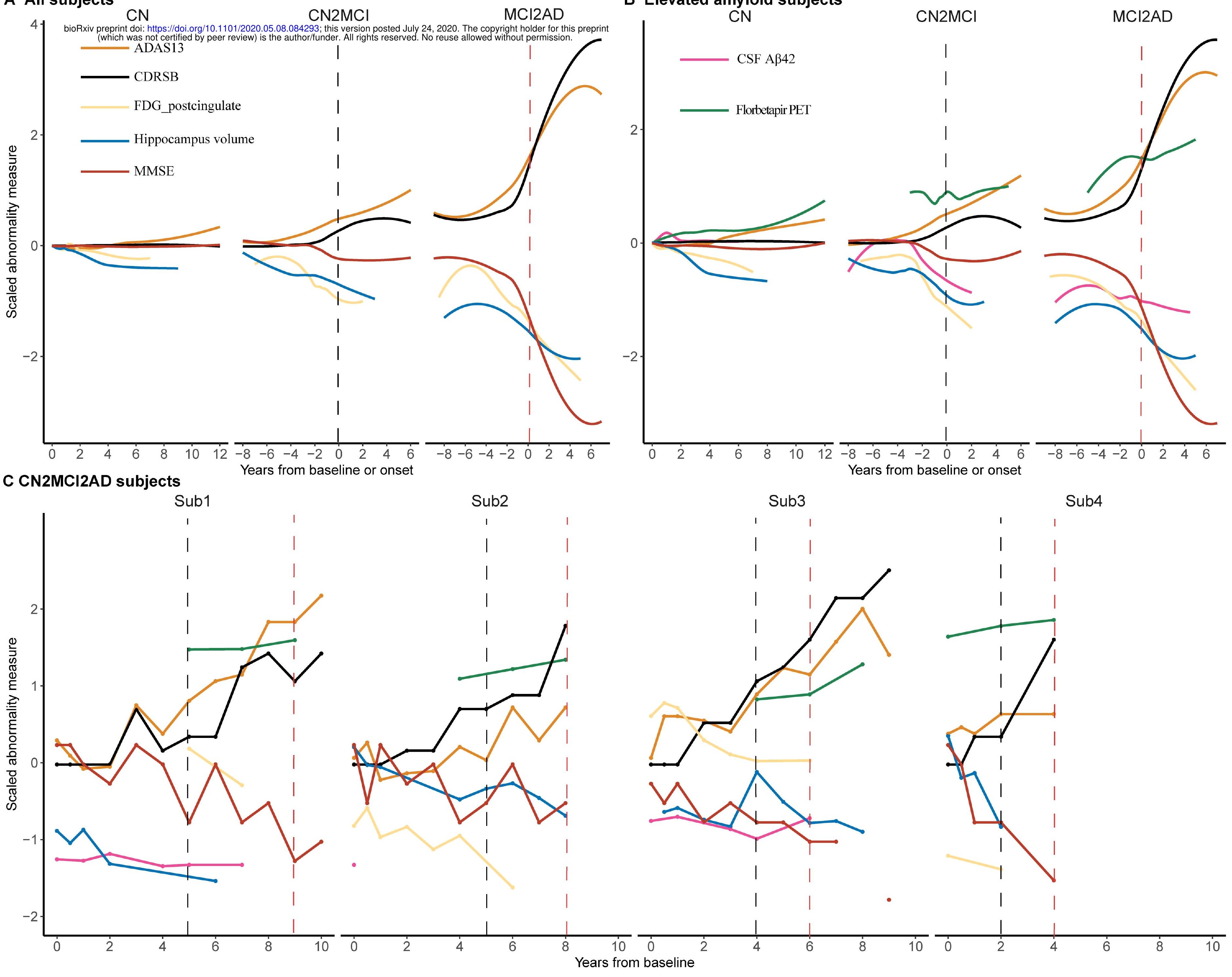

